\title{
The east London geriatric in situ simulation (ELGIS) programme: implementing in situ simulation in elderly care in two district general hospitals
}

\author{
Authors: Grace Walker," Letitia Dormandy, Corrine Quah, Rebecca Sullivan and Deblina Dasgupta
}

\begin{abstract}
Aims
To establish the feasibility and sustainability of running in situ simulation programmes on the elderly care units (ECUs) of two district general hospitals (DGHs).
\end{abstract}

\section{Methods}

We ran in situ simulation programmes at two DGHs from 2015 to 2018. Simulations were run every 2 weeks and used local simulation materials with support from the trust simulation faculty. We developed a bank of scenarios: delirium, the deteriorating multimorbid patient, falls, stroke, end-of-life care and communication scenarios around treatment escalation, nutrition and capacity. The sessions involved one trainee doctor and one nurse and were facilitated by a simulation trained senior trainee (elderly care registrar) and nurse (practice development nurse or simulation lead). Sessions lasted 45 minutes including scenario and debrief. Formal feedback was gained from participants. Latent errors were identified and acted upon via local established patient safety pathways.

\section{Results}

We conducted 42 sessions over two sites. The feedback was universally positive. Both doctors and nurses found it useful to train with colleagues: $100 \%$ of participants responded 'strongly agree' or 'agree' to the statements 'it was valuable to train with other members of the multidisciplinary team' and 'it was valuable to train in my normal environment'. A small number found it disruptive to their work. The challenges faced include the difficulty of running a scenario due to lack of beds or staff at very busy times, the dependence on specialty registrar (StR) availability and ensuring sustainability when StRs leave the trust. We developed ways to minimise these challenges. We had leadership and engagement from consultants and senior nurses to ensure simulation was seen as a priority; we had to be creative, using a day room or meeting space to run scenarios when the ward was full; and we increased

Authors: Homerton University Hospital, London, UK; * Royal College of Physicians chief registrar the number of faculty and started a simulation rota to promote the sustainability of the programme.

\section{Conclusion}

Running in situ simulation on an ECU in a DGH is feasible and popular with staff. We found that it improves interdisciplinary relationships, promotes the role of training on the unit while providing learning opportunities for juniors and teaching opportunities for senior trainees. There are challenges around sustainability and how to continue during busy periods. We have developed strategies to deal with these challenges that will be useful for other elderly care departments looking to set up in situ simulation.

\section{Conflict of interest statement}

None. 